\title{
EVALUATION OF THE MODULATORY EFFECTS OF CURCUMIN AGAINIST DOXORUBICIN-INDUCED CARDIO- AND NEPHROTOXICITIES IN RATS
}

\author{
Bakheet E. M. Elsadek, ${ }^{1}$ and Ahmed I. E. Mansour ${ }^{2}$ \\ ${ }^{1}$ Department of Biochemistry, College of Pharmacy, Al-Azhar University, Assiut Branch, P.O. \\ Box 71524 Assiut, Egypt \\ ${ }^{2}$ Department of Pharmacology and Toxicology, College of Pharmacy, Al-Azhar University, Nasr \\ City, P.O. Box 11651 Cairo, Egypt
}

ABSTRACT

The current study was designed to evaluate the modulatory effects of curcumin treatment on doxorubicin-induced nephrosis and cardiomyopathy in sprague-dawley rats. Intravenous administration of a single doxorubicin dose $(7.5 \mathrm{mg} / \mathrm{kg})$ induced apparent sings of nephrotoxicity and cardiomyopathy as manifested by marked deteriorations in both renal and cardiac parameters in association with significant changes in the histological structures of these tissues. Curcumin administration was remarkably able to mitigate the induced cardiac and kidney injuries. Moreover, it exhibited marked protective effects against such alterations. These data suggest that curcumin may have a profound therapeutic potential against such toxic effects.

\section{INTRODUCTION}

Doxorubicin, an anthracycline antibiotic, represents an extremely effective class of chemotherapeutics that have been used for the treatment of numerous malignancies since decades (Ayla et al., 2011). Doxorubicin has been used as a frontline chemotherapeutic agent used in certain human cancers including breast cancer, lung carcinoma and acute leukaemia (Mansour et al., 1999). However, the clinical application of doxorubicin is limited by some of its critical sideeffects, the most important are cardiotoxicity, haematotoxicity and a dose-limiting nephrotoxicity (Venkatesan et al., 2000; Karagiannis et al., 2010). Although the exact physiological mechanisms underlying these doxorubicin-induced cytotoxicities are not yet fully elucidated, it is widely believed that the disturbance in oxidant-antioxidant systems may be strongly implicated in such injuries through several pathways including iron-dependent oxidative damage of biological macromolecules, membrane lipid peroxidation and protein oxidation (Ayla et al., 2011).

The hypothesis was proposed that, if such induced alterations are related the oxidantantioxidant systems, then administration of the proper antioxidant therapy may greatly face against such injuries protect, or even treat, doxorubicin-related cytotoxicities.

Curcumin [1,7-bis(4-hydroxy-3-methoxyphenyl)1, 6-heptadiene-3,5-dione], the principal curcuminoid of turmeric (a traditional spice used in Indian cookery), has been recognized to exhibit beneficial role in several neurodegenerative disorders as well as treatment of other diseases (Busquets $\boldsymbol{e t}$ al., 2001; El-Sayed $\boldsymbol{e t}$ al., 2011). For instance, its administration has been reported to possess wide-range of anti-inflammatory properties. It also reduces ischaemic renal injuries, prevents renal lesions in streptozotocin diabetic rats and provides protection against oxidative stress in renal cell lines (Venkatesan et al., 2000).

Recent evidences indicate that crcumin exhibits this role through its antioxidant potential and its ability to protect against various oxidative stressors (Agrawal and Mishra 2010; Agrawal et al., 2010). Bearing all this in mind, the present study aimed to gain further insights in the modulatory effects of crcumin against doxorubicin-induced nephrotoxicity and cardiotoxicity in a Sprague-Dawley rat model. 


\section{MATERIALS AND METHODS}

\section{Materials:}

Doxorubicin hydrochloride was purchased from Yick-Vic Chemicals \& Pharmaceuticals (China), curcumin was purchased from Sigma-Aldrich Chemie GmbH (Germany), Sodium carboxy methyl cellulose was purchased from DOW Chemical Company (USA), Tween-80 was purchased from Sigma-Aldrich Chemical Co., (MO). All other chemicals and solvents were of analytical grade commercially available.

\section{Methods:}

\section{Experimental design:}

All experiments were performed using 50 healthy male Sprague-Dawley rats $(275 \pm 10 \mathrm{~g})$ obtained from from El-Neil Pharmaceutical Company, Cairo, Egypt. The animals were kept in a regulated environment (temperature, $22 \pm 1{ }^{\circ} \mathrm{C}$; humidity, $50 \pm 5 \%$; night/day cycle, 12hr) with free access to food and water ad libitum. Seven days subsequent to acclimatization of the animals, 30 rats were injected intravenously with a single dose of doxorubicin $(7.5 \mathrm{mg} / \mathrm{kg}$ body weight dissolved in $0.1 \mathrm{~mL}$ sterile normal saline) via tail vein (Pippin et al., 2009). These 30 animals were randomly divided into three groups of 10 animals each. The rats in group I were received doxorubicin only as formerly described. The rats in group II were received curcumin $(300 \mathrm{mg} / \mathrm{kg}$, orally) in a vehicle consisting of a mixture of $1 \%$ sodium carboxy methyl cellulose and $1 \%$ Tween-80 as a protective dose, 7 days before doxorubicin injection and daily thereafter throughout the study (Thiyagarajan and Sharma 2004; Isik et al., 2009). The rats in group III were received a daily dose of curcumin $(300 \mathrm{mg} / \mathrm{kg}$, orally) 10 days after doxorubicin injection for 10 days. The remaining 20 animals, that did not subjected to any doxorubicin administration, were subdivided into two groups of 10 animals each. Rats in group IV (curcumin group) were received a daily dose of curcumin $(300 \mathrm{mg} / \mathrm{kg}$, orally) from the first day of the experiment. Rats in group $\mathrm{V}$ (control group) were received a vehicle of a mixture of $1 \%$ sodium carboxy methyl cellulose and 1\% Tween-80 and therefore served as a negative control group. Animal weights were recorded every other day throughout the experimental period.

One day before the experiment termination, each animal was kept in individual metabolic cages for $24 \mathrm{hr}$ urine collection. The collected urine samples were centrifuged to remove cells and particulate material and then stored in a $-20^{\circ} \mathrm{C}$ for subsequent evaluation of the urinary biochemical parameters. On the termination day, animals were sacrificed after being fasted for 12 hours. Blood samples were collected from all animals for serum and plasma preparation which were stored at $-20^{\circ} \mathrm{C}$. Additionally, heart and both kidneys of each animal were resected, wet weights and volumes were determined and divided into two parts. The first part was stored in formalin (10\%) solution and subjected for histopathological examination and the second part was snap-frozen and stored appropriately at $-80^{\circ} \mathrm{C}$.

\section{Analytical methods:}

Serum creatinine content was assayed by kinetic procedure using the kit provided from Human Diagnostic (Germany). Blood urea nitrogen (BUN) level was assayed by colorimetric procedure using the kit provided from Biomerieux Sa (France). Serum Cystatin C contents was assayed by the radioimmunoassay procedure using the kit provided from R\&D Systems ${ }^{\circledR}$ Inc. (USA). Plasma and urine osteopontin levels were assayed by radioimmunoassay procedure using the kit provided from R\&D Systems ${ }^{\circledR}$ Inc. (USA). Total proteins contents were assayed by colorimetric procedure using the kits provided from Randox Laboratories Limited (UK). Serum creatine kinase $\mathrm{MB}$ (CK-MB), lactate dehydrogenase (LDH) and aspartate aminotransferase (AST) activities were measured by kinetic procedure using the kits provided from Reactivos GPL Barcelona (Spain). Malondialdehyde (MDA) contents, as a measure of lipid peroxidation, was spectrophotometrically measured in the renal and myocardial tissues at $532 \mathrm{~nm}$ 
using the thiobarbituric acid assay (Ohkawa et $\boldsymbol{a l}$., 1979). Levels were calculated as nmol/mg protein, while tissue proteins were determined according to the method of Lowry et al. using turbidity blank for each sample, consisting of kidney and heart homogenates added to the reagent blank (Lowry et al., 1951). Tissue levels of reduced glutathione (GSH) were measured spectrophotometrically at 412nm using Ellman assay method (Ellman and Lysko 1967). Levels were calculated as $\mathrm{nmol} / \mathrm{mg}$ protein. Superoxide dismutase (SOD) activity in renal and myocardial tissues were measured using the xanthine oxidase method as described by Sun et al. (Sun et al., 1989). Levels were calculated as U/mg protein.

\section{Histological examination}

For histopathological examination, cardiac and renal samples were fixed in $10 \%$ formalin, routinely stained with hematoxylin and eosin (H\&E) and examined through an electric light microscope.

\section{Statistical analysis}

Statistical analyses of the data were carried out using GraphPad prism version 5.0. Data comparisons were performed using analysis of variance (ANOVA). The levels of significance were accepted with $p<0.05$ and all relevant results were graphically displayed as mean \pm SEM.

\section{RESULTS AND DISCUSSION}

There is no doubt that the therapeutic application of doxorubicin as one of the most effective chemotherapeutic agents is seriously limited by the development of several side effects including mainly nephro- and cardiotoxicities. Among followed strategies to attenuate doxorubicin toxicities are dose optimization, synthesis and use of analogues or combined therapy with antioxidants in order to reduce oxidative stress without interference with its antitumor properties (Quiles $\boldsymbol{e t}$ al., 2002; El-Sayed $\boldsymbol{e t}$ al., 2011). As an example, dexrazoxane, a synthetic antioxidant drug, has been indicated for clinical use in conjunction with doxorubicin to alleviate cardiotoxicity (El-Sayed $\boldsymbol{e t}$ al., 2011). Despite its usefulness, dexrazoxane use is often limited by its interference with the antitumor efficacy of the regimen. Alternatively, certain naturally occurring substances became a focus of interest to play key roles in this indication with minimal or no side effects (El-Sayed et al., 2011). Consequently, our study was designed to examine the beneficial role of curcumin to ameliorate the renal and cardiac toxicities of doxorubicin since it is one of the well known and commonly used natural antioxidants in the traditional medicine.

Our results have shown that intravenous administration of doxorubicin produced several signs of toxicity as were manifested for example by significant decrease in body weight gain $(p<$ 0.05 ) in comparison to the healthy animals in the control group (see Table 1). This significant retardation in the animal body weights could be related to the direct toxic effects of doxorubicin on the animal intestinal mucosa as well as the indirect action on the gastrointestinal tract arising from reduced food intake causing a decrease in secretion of internal hormones and resulting in decreased trophic effects to the mucosa (Herman et al., 2000). As observed in Table 1, curcumin administration in the treated group and its co-administration in the protected group apparently attenuated doxorubicin-induced decrease in body weight with a minor superiority to the protection protocol over the treatment strategy. 
Table 1. Effect of curcumin on doxorubicin-induced changes in body weight, heart weight, heart weight to body weight percentage, kidney weight and kidney weight to body weight percentage.

\begin{tabular}{|c|c|c|c|c|c|}
\hline \multirow{2}{*}{ Parameter } & \multicolumn{5}{|c|}{ Groups } \\
\hline & Control & Doxorubicin & Curcumin & Treated & Protected \\
\hline Body weight (g) & $314.60 \pm 18.29$ & $240.00 \pm 20.55^{\dagger}$ & $325.40 \pm 17.76^{*}$ & $275.60 \pm 22.66^{\mathrm{NS}}$ & $285.60 \pm 15.40^{N S}$ \\
\hline Kidney weight (g) & $2.25 \pm 0.10$ & $1.53 \pm 0.12^{\mathrm{tt}}$ & $2.33 \pm 0.11$ ** & $1.94 \pm 0.09^{*}, \neq$ & $2.17 \pm 0.09 * *$ \\
\hline Heart weight (g) & $2.34 \pm 0.08$ & $1.57 \pm 0.09^{\mathrm{ttt}}$ & $2.32 \pm 0.08^{\star * \star}$ & $2.04 \pm 0.09^{t * * *}$ & $2.18 \pm 0.10^{* *}$ \\
\hline $\begin{array}{c}\text { Kidney:body } \\
\text { weight ratio } \times 10^{3}\end{array}$ & $7.36 \pm 0.09$ & $6.10 \pm 0.10^{t t t}$ & $7.37 \pm 0.10^{* * *}$ & $7.04 \pm 0.11^{\star \star *}$ & $7.16 \pm 0.11^{\star \star \star}$ \\
\hline $\begin{array}{l}\text { Heart:body weight } \\
\text { ratio } \times 10^{3}\end{array}$ & $7.42 \pm 0.10$ & $5.97 \pm 0.11^{\mathrm{ttt}}$ & $7.47 \pm 0.11^{\star \star \star}$ & $7.15 \pm 0.09^{* \star *}$ & $7.23 \pm 0.10^{\star \star *}$ \\
\hline
\end{tabular}

Data are presented as mean $\pm \operatorname{SEM}(\mathrm{n}=10) . \dagger, *, \ddagger$ and $\S$ indicate significant change from control group, doxorubicin group, curcumin group and treated group respectively, where NS indicates no significant difference. $\dagger, *, \ddagger$ and $\S$ indicate significant change at $P<0.05 ; \dagger \dagger, * *, \dagger \dagger$ and $\S \S$ indicate significant change at $p<0.01 ; \dagger \dagger \uparrow, * * *$, $+\uparrow \uparrow$ and $\S \S \S$ indicate significant change at $p<0.001$,

In respect to nephrotoxicity, it was clearly observed in our study that doxorubicin administration caused severe defects in the overall renal properties and functions. For instance, both the kidney weight and the ratio of kidney weight/body weight percentage were significantly lower in the doxorubicin group than in the control group $(p<0.01)$ as seen in Table 1 . These unhealthy observations were unnoticed in the two groups that received curcumin either as a curative or as protective agent. In the treated group both the kidney weight and its ratio were increased significantly with respective $\mathrm{p}$-values of $(p<0.05)$ and $(p<0.001)$ in comparison to the doxorubicin-treated group. In the protected group, curcumin co-administration produced significant increase in both the kidney weight $(p<0.01)$ and ratio $(p<0.001)$ in comparison to the doxorubicin-treated group as shown in Table 1.

Additional evidences that support these results were presented by the outcome of the biochemical indices of renal functions and histological examination of the kidney tissues which confirmed to a large extent the beneficial role of curcumin in withstanding against the doxorubicin-induced nephrotoxicity. On one hand, compared to the healthy animals of the control group, animals in the doxorubicin group suffered from marked indications of nephropathy including significant elevations in the serum Cystatin C levels $(p<0.001)$, serum creatinine levels $(p<0.001)$, circulatory BUN levels $(p<0.001)$ as well as plasma osteopontin levels $(p<$ 0.001). Moreover, severe proteinuria was pronounced in the urine of these animals with a significant increase $(p<0.001)$ in the excreted amounts of urinary proteins. As another marker of glomerular affection, a significant increase in the urinary Osteopontin excretion $(p<0.001)$ was conspicuous in the doxorubicin group in comparison to the control group (see Figure 1). All these drawbacks are in consistent with observations of several other studies and were attributed to the direct toxicities of doxorubicin towards the renal tissues (Zhang et al., 2007; Cecen et al., 2011). 

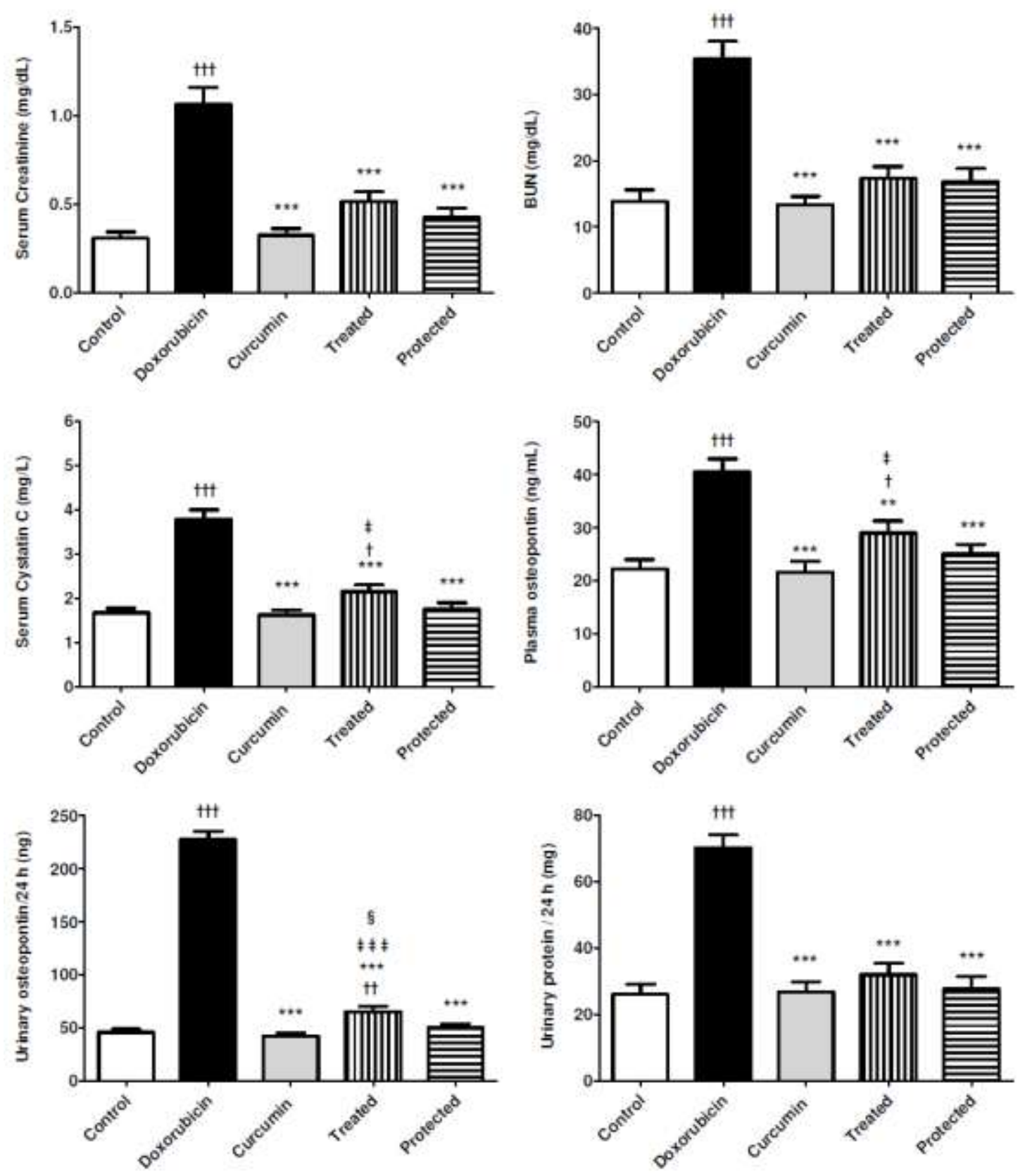

Figure 1. Effects of curcumin on doxorubicin-induced changes in the renal function biochemical indices. Data are presented as mean $\pm \operatorname{SEM}(n=10) . \dagger, *, \downarrow$ and $\S$ indicate significant change from control group, doxorubicin group, curcumin group and treated group respectively. $\dagger, *, \ddagger$ and $\S$ indicate significant change at $p<0.05 ; \dagger \dagger, * *,+\dagger$ and $\S \S$ indicate significant change at $p<0.01 ; \dagger \dagger \dagger, * * *,+\dagger+$ and $\S \S \S$ indicate significant change at $p<0.001$,

On the other hand and conversely to the doxorubicin group, curcumin administration attained significant improvements in the levels of these renal functions in both the treated and protected groups. As seen in Table 1 and Figure 1, serum Cystatin C levels were significantly decreased in both the treated $(p<0.001)$ and protected group $(p<0.001)$ and matched its corresponding levels in the healthy control group. Also, the serum creatinine levels were 
significantly reduced in both the treated $(p<0.001)$ and protected group $(p<0.001)$. Moreover, the circulatory levels of BUN were significantly normalized in both the treated $(p<0.001)$ and the protected $(p<0.001)$ group without any significant differences between any of them over the healthy control group. Furthermore, plasma levels of osteopontin, a marker of kidney injury and tissue remodeling, were significantly lower after protection $(p<0.001)$ or treatment $(p<0.001)$ with curcumin to correspond ideally to the osteopontin levels in the healthy control group.

In another confirmation for its modulatory actions, curcumin therapy was able to significantly ameliorate the doxorubicin accompanied proteinuria in both the treated and protected group. Additional substantiation for the ability of curcumin to repair the defect in the glomerular filtration was presented by the significant reduction in the urinary osteopontin excretion subsequent to curcumin co-administration in the protected group $(p<0.001)$ and Curcumin administration in the treated group $(p<0.001)$ (see Figure 1).

In the current study, evaluation of the oxidant antioxidant system introduced an additional proof for the usefulness of curcumin to modulate the doxorubicin-induced toxicities since kidneys exposed to doxorubicin alone showed aggravated signs of lipid peroxidation and oxidative stress. As shown in Figure 2, the kidneys of the animals in doxorubicin group exhibited marked imbalance in indices of lipid peroxidation as evaluated by a significant formation of thiobarbituric acid reactive substance, i. e., MDA $(p<0.001)$ with significant reductions in both the content of reduced GSH $(p<0.001)$ as well as the activity of SOD $(p<0.001)$ in comparison to the healthy animals in the control group.

In this context, curcumin was deservedly able to calm down this oxidative stress revolution and significantly reduced the MDA levels in both the treated $(p<0.001)$ and protected group $(p<0.001)$ in comparison to the doxorubicin group. Similarly, it was able to significantly refresh the doxorubicin-induced decreases in the GSH content and the SOD activity in the kidney tissues of both the treated $(p<0.001)$ and protected group $(p<0.001)$. These observations are in harmonization with several other studies that reported similar results and claimed that the presence of phenolic groups in the structure of curcumin is fundamental to explain its ability to act as a potent free radical scavenger to eliminate oxygen free radicals from the medium and that methoxy groups increase this activity. Besides, the phenolic moiety of the curcumin structure can donate hydrogen atoms to deleterious oxy radicals and form the less reactive phenoxy radicals in the process (Sreejayan and Rao 1997; Arora et al., 1998; El-Sayed et al., 2011).

Histological examinations of kidney tissues presented another strong testimony for curcumin modulatory effects to decrease the doxorubicin-induced nephrotoxicity. While, doxorubicin-adminstration induced apparent tissue alteration in the doxorubicin group in the form of focal inflammatory cells infiltration in the cortical portion, curcumin administration, on the other hand, resulted in absence of any histopathlogical alteration in the renal tissues of both the treated and protected group with normal histological structure of the glomeruli and tubules at the cortex as recorded in (Figure 3). The resultant histopathological pictures obtained after curcumin therapy were approximately identical to those observed in the renal tissues of the control group (Figure 3). This curcumin-forced attenuation of interstitial inflammation and other renal injuries could be considered as a secondary result for the inhibition of the doxorubicin induced-proteinuria (Okuda et al., 1986; Venkatesan et al., 2000). 

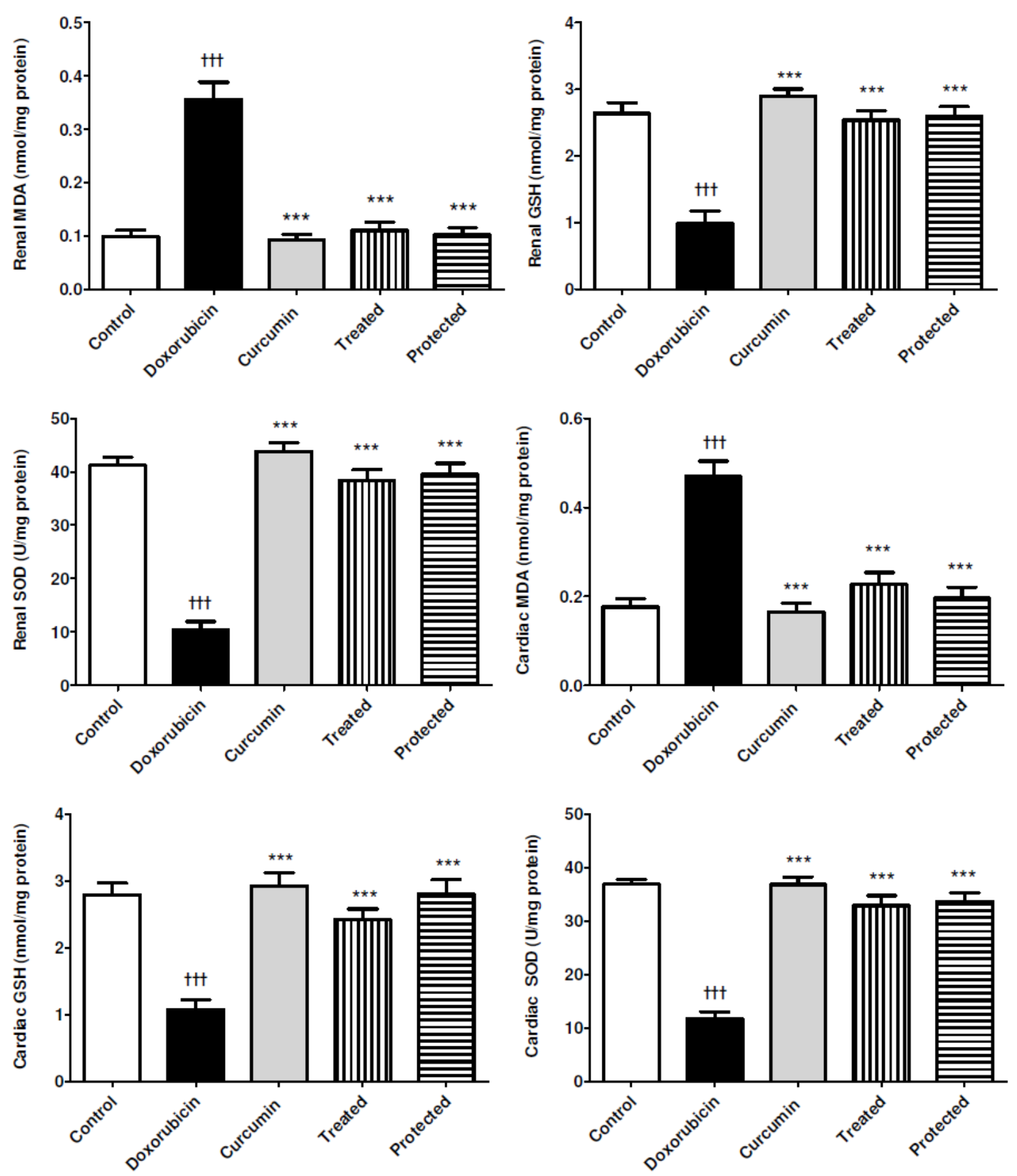

Figure 2. Effects of curcumin on doxorubicin-induced changes in the parameters of oxidative stress in both cardiac and renal tissue homogenates. Data are presented as mean \pm SEM $(\mathrm{n}=10)$. $\dagger, *,+$ and $\S$ indicate significant change from control group, doxorubicin group, curcumin group and treated group respectively. $\dagger, *, \ddagger$ and $\S$ indicate significant change at $p<0.05 ; \dagger \dagger, * *, \dagger+$ and $\S \S$ indicate significant change at $p<0.01 ; \dagger \dagger, * * *,+\uparrow+$ and $\S \S \S$ indicate significant change at $p<0.001$. 

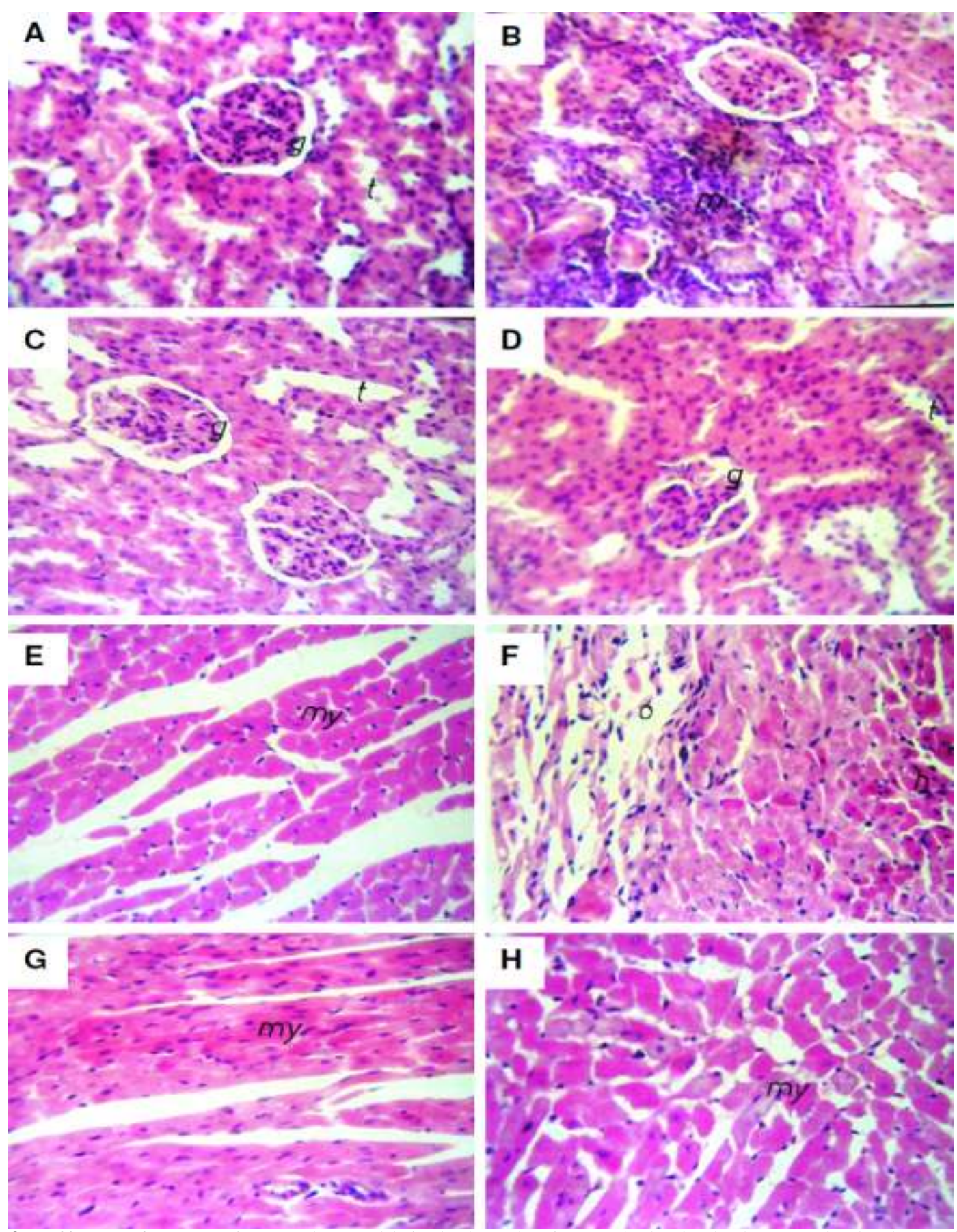

Figure 3. Effects of curcumin on doxorubicin-induced Histopathological changes in both cardiac and renal tissues. Kidney tissues in the control group [A] showed normal histological structure of the glomeruli (g) and tubules $(\mathrm{t})$ at the cortex. Conversely, kidney tissues in the doxorubicin group [B] showed focal inflammatory cells infiltration $(\mathrm{m})$ in the cortical portion. Kidneys of the treated [C] and protected groups [D] showed intact normal histological structure of the glomeruli (g) and tubules (t). Heart muscles in the control group [E] showed normal histopathlogical structure of the myocardial bundles (my). On the other hand, heart muscles in the doxorubicin group $[\mathrm{F}]$ showed apparent oedema $(\mathrm{O})$ that was dispersed the hyalinized $(\mathrm{h})$ myocardial bundles. Intermittingly, the cardiac tissues of both the treated $[\mathrm{G}]$ and protected groups $[\mathrm{H}]$ showed intact histological structure of the myocardium (my). 
Of note that, in all the aforementioned results, the modulatory effects of curcumin were more pronounced in the protected group over the treated group. The treated group showed low levels of significance than the protected group in comparison to the doxorubicin group. The only recorded significant superiority of the protected group over the treated group was obvious when curcumin administration in the treated group was significantly $(p<0.05)$ less efficient to overcome the urine osteopontin leakage than after curcumin co-administration in the protected group $(p<0.05)$.

With regard to cardiotoxicity, doxorubicin administration in the current study was implicated in several indicators of cardiac dysfunction that were manifested by significantly excessive increase in the activities of the most specific highly sensitive markers for myocardial cell injury, i. e., serum CK-MB $(p<0.001)$, LDH $(p<0.001)$ and AST $(p<0.001)$ in comparison to the healthy animals of the control group. As well, both the heart weight and the ratio of heart weight/body weight percentage were significantly decreased in the doxorubicin-treated group than in the control group $(p<0.001)$ and $(p<0.001)$ respectively indicating severe dysfunctions in cardiac performance (see Table 1). These doxorubicin-induced cardiac tissue damages are in agreement with some other result that were reported by several investigators (Venkatesan 1998; Abd-Allah et al., 2002; Bertinchant et al., 2003; Abd El-Gawad and El-Sawalhi 2004; Kelishomi et al., 2008).

The mechanism for this excessive changes and cardiac enzymes leakage seems to be from ventricular remodeling, ongoing myocyte degeneration and oxidative damage of doxorubicin to cardiac tissue with a subsequent release of its contents into circulation (DeAtley et al., 1999; Potluri et al., 2004; El-Sayed et al., 2011). This hypothesis is strongly supported by our finding that doxorubicin administration in doxorubicin group caused extreme elevations in the lipid peroxidation and markers of oxidative stress in myocardium tissues. As shown in Figure 2, intravenous doxorubicin injection resulted in a significant increase of MDA levels $(p<0.001)$ in comparison to the healthy control group. Additionally, cardiac GSH content and SOD activity in the heart muscle homogenates were significantly suppressed by doxorubicin administration.

On the contrary, curcumin administration in the treated group produced significant increase in both the heart weight $(p<0.05)$ and its ratio $(p<0.001)$ in comparison to the doxorubicin group. In the protected group, the improvements were more pronounced where curcumin co-administration produced significant increases in both the heart weight $(p<0.01)$ and its ratio $(p<0.001)$ in comparison to the doxorubicin group without any significant differences with the control group as shown in Table 1. Likewise, curcumin was found to remarkably attenuate the elevated levels of CK-MB $(p<0.001)$, LDH $(p<0.001)$ and AST $(p<0.001)$ as well as increasing the heart weight $(p<0.05)$ and its ratio $(p<0.001)$ in both the treated and protected groups in comparison to the doxorubicin group (see Figure 4). In the same respect, curcumin was able to significantly normalize the induced lipid peroxidation state in the myocardium whereas the heart muscle homogenates of both the treated and protected groups showed significantly lower levels of MDA $(p<0.001)$ and significantly higher levels of both the GSH content $(p<0.001)$ and SOD activity $(p<0.001)$ in comparison to the doxorubicin group (see Figure 2). These indicators of the curcumin-improved cardiomyopathy are in consistent with the results of other previous investigations (Wattanapitayakul et al., 2005; Srivastava and Mehta 2009). 

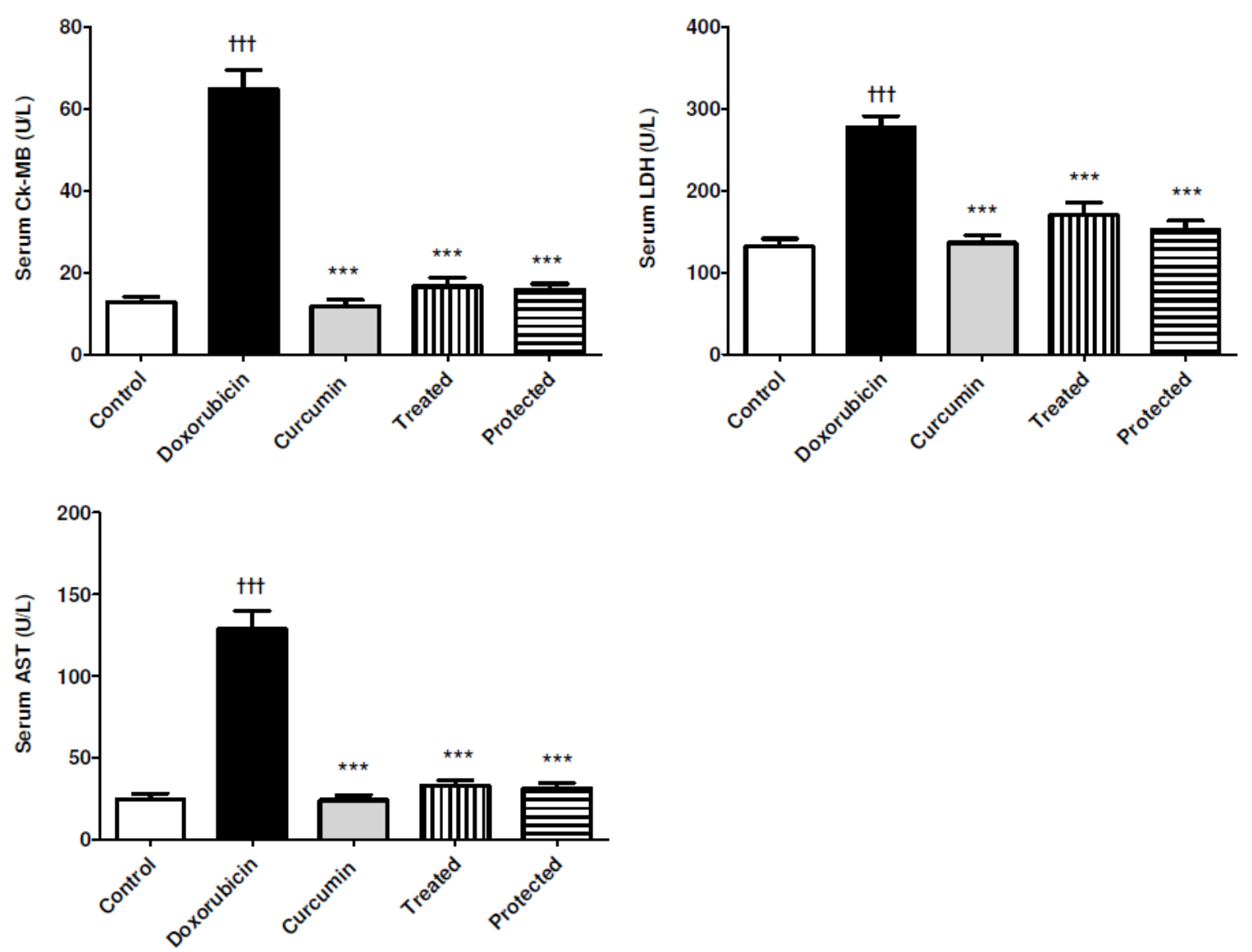

Figure 4. Effects of curcumin on doxorubicin-induced changes in the biochemical indices of myocardial dysfunction. Data are presented as mean \pm SEM $(n=10) . \dagger, *, \ddagger$ and $\S$ indicate significant change from control group, doxorubicin group, curcumin group and treated group respectively. $\dagger, *,+$ and $\S$ indicate significant change at $p<0.05 ; \dagger \uparrow, * *$, $\$+$ and $\S \S$ indicate significant change at $p<0.01$; $\dagger \dagger, * * *, \$+\$$ and $\S \S \S$ indicate significant change at $p<0.001$.

A possible explanation for these modulations could be the ability of curcumin to increase the cardiac glutathione content, suggesting that it may augment the action of these naturally occurring sulphhydryl groups to maintain membrane integrity with concomitant decrease of enzymes leakage from the cardiolcytes, protection of cardiac tissue from damage, and improvement survival of the animals (Venkatesan 1998). While the cardiac dysfunction associated with doxorubicin is attributable, at least in part, to cardiac cell apoptosis resulted from reactive oxygen species (ROS) produced by doxorubicin, some researchers have reported that agents which scavenge ROS protect against doxorubicin induced cardiac apoptosis (Kalyanaraman et al., 2002; Konorev et al., 2002). However, the majority of authors dealing with this problem considered that cardiomyopathy and nephropathy make the most important contribution to the mortality (Herman et al., 2000).

Evidences for the cardio-protective effects of curcumin are seriously augmented by our obtained histopathological investigation outcome which revealed that doxorubicin administration in the doxorubicin group leaded to hyalinization in the myocardium with oedema that was 
dispersed in between the bundles. These changes were neither observed in the control group nor in the treated or protected groups after curcumin administration that showed intact myocardium structures with no histopathlogical alterations in the myocardial bundles as recorded in (see Figure 3).

It is obvious that, the efficiency of post problematic curcumin administration in the treated group was lower than the efficiency of curcumin co-administration in the protected group but this difference was out of any statistical significance.

\section{CONCLUSIONS}

Results of the present study concluded that curcumin has multiple modulatory activities that are beneficial to protect against doxorubicin-induced toxicities to the rat kidney and heart. Therefore, its administration could be suggested as a supportive care agent during or before starting anti-cancer treatment especially containing doxorubicin. Further studies are needed in the clinical setting to determine the appropriate combination of curcumin with doxorubicin to reduce doxorubicin-induced toxicities.

\section{ACKNOWLEDGEMENTS}

The authors are grateful to Dr. Adel Bakeer Kholoussy, professor of pathology Cairo University, for carrying out the hestopathological examinations.

\section{REFERENCES}

Abd El-Gawad HM, El-Sawalhi MM. (2004): Nitric oxide and oxidative stress in brain and heart of normal rats treated with doxorubicin: role of aminoguanidine. $J$ Biochem Mol Toxicol; 18: 69-77.

Abd-Allah AR, Al-Majed AA, Mostafa AM, Al-Shabanah OA, Din AG, Nagi MN. (2002): Protective effect of arabic gum against cardiotoxicity induced by doxorubicin in mice: a possible mechanism of protection. J Biochem Mol Toxicol; 16: 254-259.

Agrawal DK, Mishra PK. (2010): Curcumin and its analogues: potential anticancer agents. Med Res Rev; 30: 818-860.

Agrawal R, Mishra B, Tyagi E, Nath C, Shukla R. (2010): Effect of curcumin on brain insulin receptors and memory functions in STZ (ICV) induced dementia model of rat. Pharmacol Res; 61: 247-252.

Arora A, Nair MG, Strasburg GM. (1998): Structure-activity relationships for antioxidant activities of a series of flavonoids in a liposomal system. Free Radic Biol Med; 24: 1355-1363.

Ayla S, Seckin I, Tanriverdi G, Cengiz M, Eser M, Soner BC, Oktem G. (2011): Doxorubicin induced nephrotoxicity: protective effect of nicotinamide. Int J Cell Biol; 2011: 390238. 
Bertinchant JP, Polge A, Juan JM, Oliva-Lauraire MC, Giuliani I, Marty-Double C, Burdy JY, Fabbro-Peray P, Laprade M, Bali JP, Granier C, de la Coussaye JE, Dauzat M. (2003): Evaluation of cardiac troponin I and $\mathrm{T}$ levels as markers of myocardial damage in doxorubicin-induced cardiomyopathy rats, and their relationship with echocardiographic and histological findings. Clin Chim Acta; 329: 39-51.

Busquets S, Carbo N, Almendro V, Quiles MT, Lopez-Soriano FJ, Argiles JM. (2001): Curcumin, a natural product present in turmeric, decreases tumor growth but does not behave as an anticachectic compound in a rat model. Cancer Lett; 167: 33-38.

Cecen E, Dost T, Culhaci N, Karul A, Ergur B, Birincioglu M. (2011): Protective effects of silymarin against doxorubicin-induced toxicity. Asian Pac J Cancer Prev; 12: 26972704.

DeAtley SM, Aksenov MY, Aksenova MV, Jordan B, Carney JM, Butterfield DA. (1999): Adriamycin-induced changes of creatine kinase activity in vivo and in cardiomyocyte culture. Toxicology; 134: 51-62.

Ellman GL, Lysko H. (1967): Disulfide and sulfhydryl compounds in TCA extracts of human blood and plasma. J Lab Clin Med; 70: 518-527.

El-Sayed E, Abd El-azeem A, Afify A, Shabana M, Ahmed H. (2011): Cardioprotective effects of Curcuma longa L. extracts against doxorubicin-induced cardiotoxicity in rats. Journal of Medicinal Plants Research; 5: 4049-4058.

Herman EH, Zhang J, Chadwick DP, Ferrans VJ. (2000): Comparison of the protective effects of amifostine and dexrazoxane against the toxicity of doxorubicin in spontaneously hypertensive rats. Cancer Chemother Pharmacol; 45: 329-334.

Isik AT, Celik T, Ulusoy G, Ongoru O, Elibol B, Doruk H, Bozoglu E, Kayir H, Mas MR, Akman S. (2009): Curcumin ameliorates impaired insulin/IGF signalling and memory deficit in a streptozotocin-treated rat model. Age (Dordr); 31: 39-49.

Kalyanaraman B, Joseph J, Kalivendi S, Wang S, Konorev E, Kotamraju S. (2002): Doxorubicin-induced apoptosis: implications in cardiotoxicity. Mol Cell Biochem; 234-235: 119-124.

Karagiannis TC, Lin AJ, Ververis K, Chang L, Tang MM, Okabe J, El-Osta A. (2010): Trichostatin A accentuates doxorubicin-induced hypertrophy in cardiac myocytes. Aging (Albany NY); 2: 659-668.

Kelishomi RB, Ejtemaeemehr S, Tavangar SM, Rahimian R, Mobarakeh JI, Dehpour AR. (2008): Morphine is protective against doxorubicin-induced cardiotoxicity in rat. Toxicology; 243: 96-104.

Konorev EA, Kotamraju S, Zhao H, Kalivendi S, Joseph J, Kalyanaraman B. (2002): Paradoxical effects of metalloporphyrins on doxorubicin-induced apoptosis: scavenging of reactive oxygen species versus induction of heme oxygenase-1. Free Radic Biol Med; 33: 988.

Lowry OH, Rosebrough NJ, Farr AL, Randall RJ. (1951): Protein measurement with the Folin phenol reagent. J Biol Chem; 193: 265-275. 
Mansour MA, El-Kashef HA, Al-Shabanah OA. (1999): Effect of captopril on doxorubicininduced nephrotoxicity in normal rats. Pharmacol Res; 39: 233-237.

Ohkawa H, Ohishi N, Yagi K. (1979): Assay for lipid peroxides in animal tissues by thiobarbituric acid reaction. Anal Biochem; 95: 351-358.

Okuda S, Oh Y, Tsuruda H, Onoyama K, Fujimi S, Fujishima M. (1986): Adriamycin-induced nephropathy as a model of chronic progressive glomerular disease. Kidney Int; 29: $502-510$.

Pippin JW, Brinkkoetter PT, Cormack-Aboud FC, Durvasula RV, Hauser PV, Kowalewska J, Krofft RD, Logar CM, Marshall CB, Ohse T, Shankland SJ. (2009): Inducible rodent models of acquired podocyte diseases. Am J Physiol Renal Physiol; 296: F213-229.

Potluri S, Ventura HO, Mulumudi M, Mehra MR. (2004): Cardiac troponin levels in heart failure. Cardiol Rev; 12: 21-25.

Quiles JL, Huertas JR, Battino M, Mataix J, Ramirez-Tortosa MC. (2002): Antioxidant nutrients and adriamycin toxicity. Toxicology; 180: 79-95.

Sreejayan, Rao MN. (1997): Nitric oxide scavenging by curcuminoids. J Pharm Pharmacol; 49: 105-107.

Srivastava G, Mehta JL. (2009): Currying the heart: curcumin and cardioprotection. $J$ Cardiovasc Pharmacol Ther; 14: 22-27.

Sun L, Peterson TE, McCormick ML, Oberley LW, Osborne JW. (1989): Improved superoxide dismutase assay for clinical use. Clin Chem; 35: 1265-1266.

Thiyagarajan M, Sharma SS. (2004): Neuroprotective effect of curcumin in middle cerebral artery occlusion induced focal cerebral ischemia in rats. Life Sci; 74: 969-985.

Venkatesan N. (1998): Curcumin attenuation of acute adriamycin myocardial toxicity in rats. $\mathrm{Br}$ J Pharmacol; 124: 425-427.

Venkatesan N, Punithavathi D, Arumugam V. (2000): Curcumin prevents adriamycin nephrotoxicity in rats. Br J Pharmacol; 129: 231-234.

Wattanapitayakul SK, Chularojmontri L, Herunsalee A, Charuchongkolwongse S, Niumsakul $S$, Bauer JA. (2005): Screening of antioxidants from medicinal plants for cardioprotective effect against doxorubicin toxicity. Basic Clin Pharmacol Toxicol; 96: 80-87.

Zhang Y, Li J, Liu KJ. (2007): [Effect of Kangxianling on Smads signal pathway molecules in rats with adriamycin induced nephropathy]. Zhongguo Zhong Xi Yi Jie He Za Zhi; 27: 1094-1098. 
تقييم فاعلية الكركمين في مقاومة إعتلال الكلى وعضلة القلب الناجمين عن تعاطي عقار الدوكسوروبيسين في نموذج الفئران

$$
\begin{aligned}
& \text { 'بخيت القط مصطفى- 'أحمد محمد إبراهيم منصور }
\end{aligned}
$$

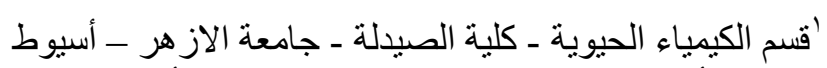

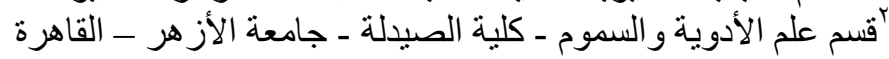

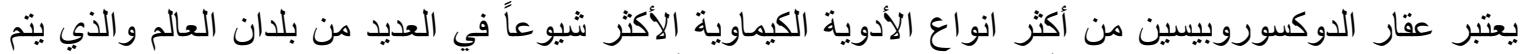

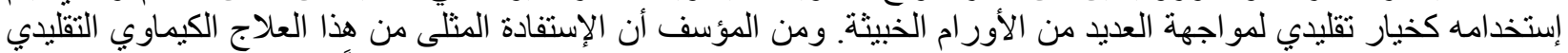

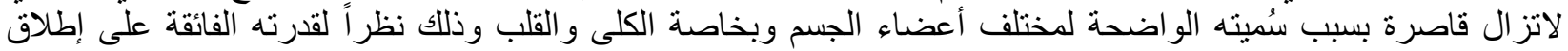

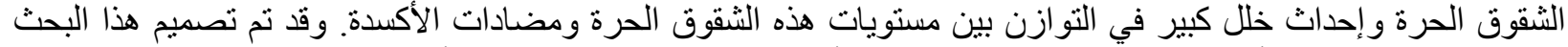

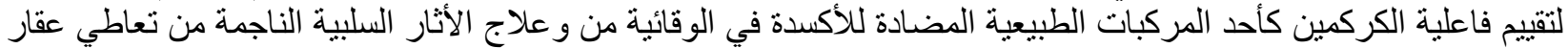

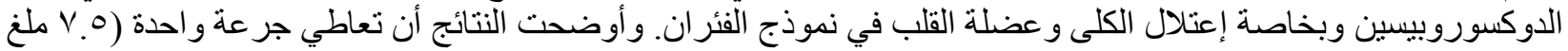

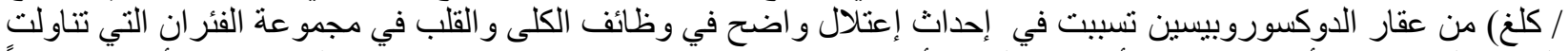

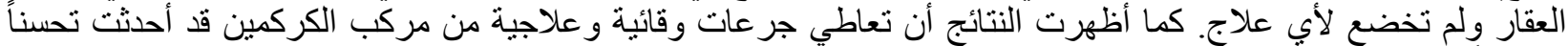

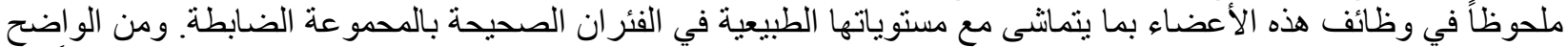

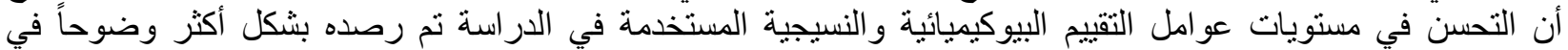

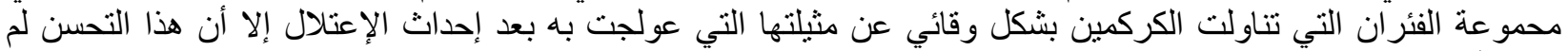

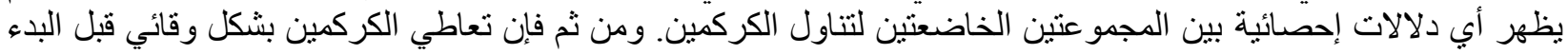

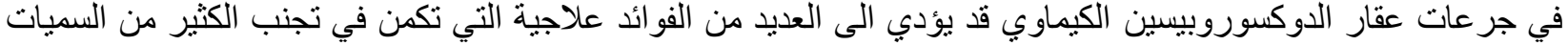

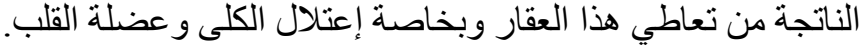

